\title{
Accuracy of Triggering Receptor Expressed on Myeloid Cells 1 in diagnosis and prognosis of acute myocardial infarction: a prospective cohort study
}

\author{
Zhenjun Ji ${ }^{1}$, Rui Zhang ${ }^{1}$, Mingming Yang ${ }^{1}$, Wenjie Zuo ${ }^{1}$, Yuyu Yao ${ }^{1}$, Yangyang Qu ${ }^{1}$, Yamin Su ${ }^{1}$, Zhuyuan Liu ${ }^{1}$, \\ Ziran Gu ${ }^{1}$, Genshan Ma ${ }^{\text {Corresp. } 1}$ \\ ${ }^{1}$ Department of Cardiology, Zhongda Hospital, School of Medicine, Southeast University, Nanjing, Jiangsu, China \\ Corresponding Author: Genshan Ma \\ Email address: 101010771@seu.edu.cn
}

Background. Acute myocardial infarction (AMI) is one of the fatal cardiac emergencies. The detection of Triggering receptor expressed on myeloid cells 1 (TREM1), a cell surface immunoglobulin that amplifies pro-inflammatory responses, screened by bioinformatics was shown to be significant in diagnosing and predicting the prognosis of AMI.

Methods. GSE66360, GSE61144, and GSE60993 were downloaded from the Gene Expression Omnibus (GEO) database to explore the differentially expressed genes (DEGs) between AMI and control groups using R software. 147 patients in total were prospectively enrolled from October 2018 to June 2019 and divided into two groups, the normal group $(n=35)$ and the AMI group $(n=112)$. Plasma was collected from each patient at admission and all patients received six-month follow-up care.

Results. According to bioinformatic analysis, TREM1 was an important DEG in patients with AMI. Compared with the normal group, TREM1 expression was markedly increased in the AMI group $(p<0.001)$. TREM1 expression was positively correlated with fasting plasma glucose (FPG), glycosylated hemoglobin ( $\mathrm{HbAC})$, and the number of lesion vessels, although it had no correlation with Gensini score. TREM1 expression in the triple-vessels group was significantly higher than that of the single-vessel group $(p<0.05)$. Multiple linear regression showed that UA and HbAC were two factors influencing TREM1 expression. The ROC curve showed that TREM1 had a diagnostic significance in $\mathrm{AMI}(\mathrm{P}<0.001)$, especially in AMI patients without diabetes. Cox regression showed increased TREM1 expression was closely associated with six-month major adverse cardiac events (MACEs) $(P<0.001)$.

Conclusions. TREM1 is a potentially significant biomarker for the diagnosis of AMI and may be closely associated with the severity of coronary lesions and diabetes. TREM1 may also be helpful in predicting the six-month MACEs after AMI. 
1 Accuracy of Triggering Receptor Expressed on Myeloid Cells 1 in diagnosis and prognosis of acute myocardial infarction: a prospective cohort study

\section{Corresponding author:}

\section{Abstract} six-month follow-up care.

*Genshan Ma, MD, PhD, Professor.

Department of Cardiology, Zhongda Hospital, School of Medicine, Southeast University, Dingjiaqiao 87, Hunan Road, Nanjing, Jiangsu, China.

\section{Email address: 101010771@seu.edu.cn.}

Background. Acute myocardial infarction (AMI) is one of the fatal cardiac emergencies. The detection of Triggering receptor expressed on myeloid cells 1 (TREM1), a cell surface immunoglobulin that amplifies pro-inflammatory responses, screened by bioinformatics was shown to be significant in diagnosing and predicting the prognosis of AMI.

Methods. GSE66360, GSE61144, and GSE60993 were downloaded from the Gene Expression Omnibus (GEO) database to explore the differentially expressed genes (DEGs) between AMI and control groups using $\mathrm{R}$ software. 147 patients in total were prospectively enrolled from October 2018 to June 2019 and divided into two groups, the normal group $(n=35)$ and the AMI group ( $\mathrm{n}=112$ ). Plasma was collected from each patient at admission and all patients received

Results. According to bioinformatic analysis, TREM1 was an important DEG in patients with AMI. Compared with the normal group, TREM1 expression was markedly increased in the AMI group $(\mathrm{p}<0.001)$. TREM1 expression was positively correlated with fasting plasma glucose (FPG), glycosylated hemoglobin (HbAC), and the number of lesion vessels, although it had no correlation with Gensini score. TREM1 expression in the triple-vessels group was significantly higher than that of the single-vessel group $(\mathrm{p}<0.05)$. Multiple linear regression showed that UA and $\mathrm{HbAC}$ were two factors influencing TREM1 expression. The ROC curve showed that TREM1 had a diagnostic significance in AMI $(\mathrm{P}<0.001)$, especially in AMI patients without diabetes. Cox regression showed increased TREM1 expression was closely associated with sixmonth major adverse cardiac events (MACEs) $(\mathrm{P}<0.001)$. 
35 Conclusions. TREM1 is a potentially significant biomarker for the diagnosis of AMI and may be closely associated with the severity of coronary lesions and diabetes. TREM1 may also be helpful in predicting the six-month MACEs after AMI.

Key words TREM1, myocardial infarction, diagnosis, prognosis, bioinformatics

\section{Introduction}

The mortality rates of cardiovascular diseases continues to rise in Chinese cities and rural areas (Hu SS, 2018). As one of the most important cardiovascular emergencies, acute myocardial infarction (AMI) is generally divided into ST-segment elevated myocardial infarction (STEMI) and Non-STEMI (NSTEMI) (Thygesen et al., 2019). Early diagnosis and treatment are critical to reducing the incidence of major adverse cardiac events (MACEs) in AMI patients. Currently, clinical diagnosis of AMI still depends on the dynamic change of myocardial infarction (MI) biomarkers and electrocardiogram (ECG). The outcome of patients with AMI is usually predicted by age, left ventricular function, the severity of coronary artery stenosis, and myocardial ischemia (Loscalzo, 2013). Hence, it is important to explore new effective biomarkers for the diagnosis and prognosis of AMI.

The rise and development of genomics, proteomics, metabolomics, and transcriptomics have created a large amount of data for clinical and basic research. Bioinformatics methods can be used for secondary analysis and processing of existing bioinformatics resources, such as gene function annotation, pathway analysis, and enrichment analysis, to better carry out life science research. We searched existing blood sequencing datasets of patients with AMI in Pubmed and screened the appropriate differentially expressed genes (DEGs) using bioinformatics methods. Combined with the latest literature progress, Triggering receptor expressed on myeloid cells 1 (TREM1) and Toll-like receptor 4 (TLR4) were finally screened as the important DEGs between patients with AMI and healthy controls, and verified them in clinical samples.

The TREM1 gene is located on human chromosome 6 and expressed as two isoforms: membrane-bound TREM1 and soluble TREM1 (sTREM1) (Jeremie et al., 2015). TREM1 is an immune receptor expressed in human neutrophils, monocytes, and macrophages; its expression may be related to the activation of Toll-like receptors (TLR) (Jeremie et al., 2015). Amir Boufenzer et al (2015) studied the role of TREM1 in AMI based on animal models, which showed that inhibiting TREM1 activation may reduce the inflammatory response after myocardial infarction. Furthermore, they also reported that a new synthetic short peptide, LR12, 
69 can inhibit the expression of TREM1 and reduce neutrophil aggregation, monocyte chemokine

70 production, and improve cardiac function after myocardial infarction in mice and rats (Boufenzer

71 et al., 2015). Anton G. Kutikhin et al (2016) found that specific TLR was associated with the

72 polymorphism of the TREM1 gene (including the C/T genotype of TLR4 rs4986791) and the

73 severity of atherosclerosis in Russian population. TREM1 mediated pathways such as the IL2

74 pathway, MHC I-mediated APC, and the Toll-like receptors pathway were all involved in the

75 process of innate immunity inflammation after the onset of AMI (Ormsby et al., 2011; Tessarz \&

76 Cerwenka, 2008). However, the significance of TREM1 for guiding clinical diagnosis and

77 treatment of cardiovascular disease still requires further study.

This study aims to explore the role of TREM1 in the diagnosis of MI and prediction of major adverse cardiac events (MACEs).

Materials and methods

83 Screening DEGs

84 The data expression files GSE66360, GSE61144, and GSE60993 were screened and downloaded from the Gene Expression Omnibus (GEO) database (http:/www.ncbi.nlm.nih.gov/geo). The GSE61144 dataset, including 7 patients with STEMI and 10 patients with normal control, aimed to identify early significant serum biomarkers of STEMI. GPL6106 Sentrix Human-6 v2 Expression BeadChip was the sequencing platform for GSE61144. The GSE66360 dataset, including 49 patients with STEMI and 50 healthy cohorts, aimed to present the specific gene expression pattern in whole blood of patients and utilized the HG-133U_PLUS_2 microarray sequencing platform. The GSE60993 dataset, including 7 STEMI patients and 7 healthy patients, aimed to study the molecular signature of acute coronary syndrome (ACS) based on blood transcriptome and to identify novel serum biomarkers for early-stage STEMI. The Illumina HumanWG-6 v3.0 expression BeadChip sequence platform was used for GSE60993. The affyPLM package was applied for data quality control. GSE61144/66360/60993 were treated with limma package in R software to explore the differentially expressed genes (DEGs) between STEMI and control groups. The threshold of differential genes had a false discovery rate (FDR) $<0.05$ and $\mid \operatorname{logFCl}>1$. A Venn diagram drawn using an online tool

99 (http://bioinformatics.psb.ugent.be/webtools/Venn/) generated the common genes intersection of 100 different datasets. Protein-protein interaction (PPI) network construction was performed for 101 functional proteins association analysis using String database (http://string-db.org/), which 
102 predicted the physical and functional association among specific proteins. cytoHubba plugin in

103 Cytoscape (https://cytoscape.org/) was used for node network topology analysis of PPI network

104 and pivotal nub genes were screened according to Degree.

105

\section{Study design}

107 This study was a prospective, case-control, and cohort study. Patients with AMI or with normal

108 coronary arteries were enrolled and prospectively followed up for six months to study the 109 diagnostic and prognostic roles of TREM1.

110

\section{Setting}

112 The study was approved by the Ethics Committee of Zhongda Hospital Southeast University 113 (2018ZDSYLL134-P01). All patients gave written informed consents. Patients with AMI or 114 normal coronary arteries were consecutively enrolled from October 2018 to June 2019 in 115 Zhongda Hospital Southeast University according to the inclusion and exclusion criteria. 116 Laboratory and imaging examination data were collected. All enrolled patients received at least 117 six-months follow-up.

\section{Participants}

120 In total, 147 patients were enrolled and divided into the normal group $(n=35)$ and the AMI group 121 ( $\mathrm{n}=112)$. Inclusion criteria: 1. Willing to participate in the study and giving informed consent; 2.

122 Male or non-pregnant female aged 18-85; 3. Normal group: patients with normal coronary 123 arteries confirmed by coronary angiography (CAG). AMI group: Typical chest pain, elevated 124 TnI, and ECG showed ischemic ST-T changes. Those who met all the criteria above were 125 selected. Exclusion criteria: 1. Previous coronary artery bypass grafting history; 2. Severe liver 126 or kidney disease (not caused by heart disease); 3. Uncontrolled severe hypertension (systolic 127 blood pressure $>180 \mathrm{mmHg}$ and diastolic blood pressure $>100 \mathrm{mmHg}$ after standard treatment); 4 .

128 Ischemic stroke within one week, previous history of intracranial hemorrhage, gastrointestinal 129 bleeding within six months, or major surgery within 30 days; 5. Severe dyspnea, such as 130 bronchial asthma and chronic obstructive pulmonary disease; 6. Suffering from other severe 131 diseases and having a life expectancy less than half a year; 7. Pathological sinus node syndrome,

132 Grade II or III atrioventricular block, or previous syncope history due to bradycardia; 8. Pregnant

133 or lactating women; 9. Other circumstances, as determined by the researchers, which would have 134 made the patient unsuitable for participation in this study. Any patients who did not meet any 135 exclusion criteria were selected. The flow chart was shown in Figure 1. 


\section{Variables and data sources}

138 Basic laboratory and imaging results included routine blood tests, blood biochemistry tests, 139 fibrinolytic testing, and echocardiography. Basic characteristics included age, gender, history of 140 smoking, diabetes and hypertension, height and weight, and systolic blood pressure. Count of red 141 blood cells (RBC) and white blood cells (WBC), hemoglobin ( $\mathrm{Hb}$ ), alanine aminotransferase 142 (ALT), aspartate aminotransferase (AST), total cholesterol (TC), total triglyceride (TG), albumin 143 (ALB), bilirubin, blood urea nitrogen (BUN), uric acid (UA), fasting plasma glucose (FPG), 144 glycosylated hemoglobin (HbAC), C-reactive protein (CRP), BNP, and left ventricular ejected 145 fraction (LVEF) were all collected. The enrolled MI patients were divided into single-vessel, 146 double-vessels, and triple-vessels groups according to the number of lesion vessels with $>50 \%$ 147 stenosis in the left main, left anterior descending, left circumflex, and right coronary arteries. 148 Gensini score calculation was performed following Gensini's (1983) published guidelines.

149 Follow-up major adverse cardiovascular events include recurrent arrhythmia, target vessel 150 revascularization (TVR), recurrent myocardial infarction, cardiogenic death, and all-cause death 151 (Kip et al., 2008).

152

\section{Bias}

154 Bias is a systematic error caused by the fact that the results obtained from the comparative study 155 of each sample group cannot truthfully reflect the real results in the target population. For the 156 systematic error in the selection of study objects-selection bias, we used the medical records 157 system, telephone, and other methods for the follow-up of all patients, to avoid loss of follow-up 158 and reduce bias as far as possible.

159 We selected the control group from the cardiology department at the same time as the AMI 160 patients in order to reduce differences between the two groups. For the information bias, which 161 mainly occurs in the implementation stage of observation and measurement, we used 162 quantifiable objective indicators as far as possible, while excluding the information bias caused 163 by subjective guesswork. Statistical machines and operating procedures were used in laboratory 164 testing and enzyme-linked immunosorbent assay (ELISA) testing to avoid large information bias. 165 To reduce the confounding biases that can occur in both the design and analysis phases, 166 multivariate analysis and Cox regression were used during data analysis for statistical correction.

\section{Study size}


169 The clinical sample size was calculated using PASS15.0 software. Since we could not consult the

170 relevant literature on the expression of TREM1 in plasma, we adjusted the sample size through

171 the pre-experiment. The TREM1 expression in the normal group was $127.1 \pm 50.1 \mathrm{pg} / \mathrm{ml}(\mathrm{n}=16)$

172 and in the AMI group was 180.2 $\pm 70(\mathrm{n}=60)$ (Table S1). According to the control: case $=1: 4$

173 (Two-Sample T-Tests) and taking the rate of loss of follow-up $<20 \%$ into consideration, it was

174 calculated to include 113 cases. Since TREM1 indicators are rarely reported in the clinical

175 literature, and to further reduce bias, we eventually included 147 samples.

176

\section{Sample collection and the enzyme-linked immunosorbent assay (ELISA)}

$1785 \mathrm{~mL}$ of peripheral blood was collected from each patient within 4 hours of admission. Blood 179 samples were collected by EDTA anticoagulant tubes, stored in refrigerators at $4^{\circ} \mathrm{C}$, and 180 processed within 2 hours. The samples were centrifuged at $3000 \mathrm{r} / \mathrm{min}$ and $4^{\circ} \mathrm{C}$ for $30 \mathrm{~min}$. The 181 plasma was collected and stored in $-80^{\circ} \mathrm{C}$ refrigerators. ELISA was performed by TREM1 Elisa

182 kit (CUSABIO, China). Anti-TREM1 was coated on the enzyme-label plates. 100 $\mu 1$ plasma was 183 added into each well and incubated at $37^{\circ} \mathrm{C}$ incubator (JINGHONG, Shanghai) for 1 hour. 184 Afterwards, the plates were washed with phosphate buffered saline tween-20 (PBST) 5 times, 18530 s each time. Then $100 \mu \mathrm{l}$ Biotin-antibody was added into each well and incubated at $37^{\circ} \mathrm{C}$ for 1 186 hour, and then washed for another $30 \mathrm{~s} \times 3$ times. Next, we added $100 \mu \mathrm{HRP}$-avidin to each well 187 which was incubated again for 1 hour at $37^{\circ} \mathrm{C}$. $90 \mu \mathrm{TMB}$ Substrate was added into each well and 188 incubated for $30 \mathrm{~min}$ at $37^{\circ} \mathrm{C}$ in a dark environment. Then $50 \mu 1$ Stop Solution was added into each 189 well, which was read using Microplate Reader (RAYTO RT-6000, Shenzhen) at 450nm within $1905 \mathrm{~min}$.

191

\section{Statistical methods}

193 Data was processed by SPSS 23.0 and GraphPad Prism 7.0. T-test was used for comparison 194 between two groups and Anova analysis was used for comparison of multiple groups. Pearson 195 correlation analysis and multiple linear regressions were performed to analyze factors affecting 196 variables. Univariate and variate Cox regressions were used for survival and prognosis studies. If 197 the data did not satisfy normality and homogeneity of variance $(\mathrm{SD} \geq \chi)$, median and quartile $198(25 \%, 75 \%)$ were calculated. Variables included in the variate regression were either confirmed 199 clinically relevant or validated by univariate analysis.

200

201 Results

202 Candidate genes from screening and PPI network construction 
203 323, 128, and 147 significantly up-regulated DEGs were respectively screened out in GSE66360, 204 GSE61144, and GSE60993 datasets where 86, 42, and 25 down-regulated DEGs were also found, 205 respectively. The data was graphed into volcano plots (Fig. 2). After the three datasets were 206 integrated, we found 29 common up-regulated and 3 down-regulated genes (Table 1). The final 20732 screened genes were submitted to String database to construct an interaction network (Fig. 2), 208 and then cytoHubba was used to analyze hub genes. TLR4 (Degree:11.0), TREM1 (Degree:9.0) 209 and S100A12 (Degree:9.0) were three important up-regulated genes. Previous studies have 210 reported that S100A12 was an important inflammatory marker in coronary heart disease 211 (Ligthart $S$ et al., 2014; Wang X et al., 2019), therefore, TLR4 and TREM1 were further studied.

212

$213 \quad$ Basic characteristics of patients

214147 patients were included and completed follow-up. During the half-year follow-up, 48 events

215 happened in AMI patients. There was significant difference in smoking $(\mathrm{p}<0.001)$ between two 216 groups. Compared with the normal group, there were higher age, levels of white blood cells 217 (WBC), and fasting plasma glucose $(F P G)$ in the AMI groups $(p<0.05)$, while left ventricular 218 ejected fraction (LVEF) and albumin (ALB) were significantly decreased $(\mathrm{p}<0.005)$. The levels 219 of ALT and AST were significantly higher in the AMI group than the normal group $(\mathrm{p}<0.001)$. 220 The levels of LDH and CK and DDimer were also measurably higher in the AMI group (Table 221 2).

222

Differential expression of TREM1 and Pearson analysis According to the bioinformatic results, we examined the expression of TREM1 and TLR4 (Table S1). The result showed no significant difference of TLR4 between two groups. Compared with the normal group, TREM1 expression was markedly increased in the AMI group ( $<<0.001)$ (Fig. 3A). Pearson correlation analysis showed that TREM1 expression was positively correlated with age ( $\mathrm{r}=0.222, \mathrm{P}=0.007)$, neutrophil $(\mathrm{r}=0.209, \mathrm{P}=0.011), \mathrm{BUN}(\mathrm{r}=0.253, \mathrm{P}=0.003), \mathrm{UA}(\mathrm{r}=0.335$, $\mathrm{P}<0.001)$, FPG $(\mathrm{r}=0.279, \mathrm{P}=0.001)$, HbAC $(\mathrm{r}=0.379, \mathrm{P}<0.001), \mathrm{BNP}(\mathrm{r}=0.411, \mathrm{P}<0.001)$, and left atrial diameter $(\mathrm{r}=0.212, \mathrm{P}=0.014)$, and negatively correlated with ALB $(\mathrm{r}=-0.187, \mathrm{P}=0.024)$ and ApoA1 ( $\mathrm{r}=-0.167, \mathrm{P}=0.046)$ (Table 3). TREM1 expression in the blood was not correlated with monocytes number $(r=0.084, \mathrm{P}=0.309)$ and neutrophil-lymphocyte ratio (NLR) $(\mathrm{r}=0.128$, $\mathrm{P}=0.121$ ). Variables with great significance in Pearson analysis were included in multiple linear 234 regression. Due to the known correlation between $\mathrm{HbAC}$ and FPG, HbAC representing the level 235 of blood glucose control within 2-3 months was selected as one of the regression variables. 236 Gensini score $(\mathrm{P}=0.327)$ was also selected as a variable because of its significance representing 
237 the severity of coronary lesions. Finally, age, neutrophil, BUN, UA, HbAC, BNP, 238 left atrial diameter, ALB, ApoA1, lesion vessels, and Gensini score were included in the 239 regression. Three methods of linear regression, Stepwise, Forward, and Backward, were all run 240 in SPSS, which showed that BNP and HbAC were two important independent factors influencing 241 TREM1 expression. The fitting equation: Ý(TREM1) $=0.035 * \mathrm{BNP}+16.520 * \mathrm{Hb} \mathrm{AC}+66.233$ $242(\mathrm{R} 2=0.283, \mathrm{~F}=7.905, \mathrm{P}=0.001)$. When $\mathrm{BNP}$ are addressed, TREM1 was increased by 16.520 243 units for every one unit increase in HbAC (Table 3).

244

TREM1 and subgroup analysis

246 TREM1 was positively correlated with lesion vessels $(\mathrm{r}=0.244, \mathrm{P}=0.011)$, although it had no 247 correlation with Gensini score $(p=0.327)$. TREM1 expression in the triple-vessels group $248(223.9 \pm 101.0 \mathrm{pg} / \mathrm{ml})$ was significantly higher than that of the single-vessel group $(172.5 \pm 74.5$ $249 \mathrm{pg} / \mathrm{ml})(\mathrm{P}<0.001)$; the level in the double-vessels group was 202.7 \pm 77.3 (pg/ml) (Fig. 3B).

250 TREM1 expression showed a significant difference in the normal and AMI groups in diabetes 251 subgroups (Fig. 3C). Compared to the normal group without diabetes, TREM1 expression was 252 higher in the AMI group with or without diabetes $(p<0.05)$. In the AMI group, TREM1 253 expression was higher in patients with diabetes than the group without diabetes, although it 254 showed no significant difference $(\mathrm{p}=0.06)$.

255

TREM1 and diagnosis of AMI

The ROC curve showed that TREM1 played a novel role in the diagnosis of AMI $(\mathrm{P}<0.001)$ when the area under the curve (AUC) was 0.721 , with a sensitivity of 0.786 and a specificity of 0.600 (Yoden Index =0.386) (Fig. 4A). In AMI groups without diabetes, the diagnostic sensitivity was 0.757 , the specificity was 0.631 and the AUC was 0.724 (Yoden Index $=0.402$, $\mathrm{P}<0.001$ ) (Fig. 4B). It seemed that TREM1 showed more significant diagnostic value in AMI patients without diabetes mellitus.

263

TREM1 and prognosis of AMI

The role of TREM1 in the six-month follow-up was also studied. MACEs such as rehospitalization because of recurrent angina pectoris and heart failure, revascularization, recurrent MI, cardiac death, and total death were all recorded. Patients with AMI were divided into high risk and low risk groups according to the median of TREM1 (high risk $\geq 177.5$, low risk 
271 performed to explore the important variables affecting MACE incidences (Table 4). TREM1

$272(\mathrm{p}<0.001)$, heartrate $(\mathrm{p}=0.001), \mathrm{RBC}(\mathrm{p}=0.048), \mathrm{Hb}(\mathrm{p}=0.019), \mathrm{CK}(\mathrm{p}=0.022), \mathrm{FPG}(\mathrm{p}=0.021)$,

273 BUN ( $p=0.013)$, UA $(p=0.044)$, LA ( $p=0.05)$ IVS $(p=0.031), \operatorname{LV}(p=0.020), \operatorname{LVEF}(p=0.001)$,

274 and vessels $(\mathrm{p}=0.040)$ screened by univariate Cox regression analysis were entered into the

275 variate Cox analysis. Finally, we determined that TREM1, Hb and IVS were three important

276 independent factors affecting the six-month outcome of AMI patients $(\mathrm{F}=34.091, \mathrm{P}<0.001)$.

277

\section{Discussion}

279 We used bioinformatics to search gene expression profiles of AMI to explore the DEGs of AMI.

280 According to Cytoscape, TREM1 and TLR4 were considered two of the most important genes of

281 AMI, but only TREM1 showed a significant difference. We found that TREM1 may be helpful

282 for the diagnosis of MI and is associated with the number of lesion vessels. The more novel

283 findings were that TREM1 was closely associated with diabetes mellitus and it was an

284 independent predictor for six-month MACEs after AMI. There was higher incidence of MACEs

285 in patients with a higher TREM1 level.

287 As a transmembrane glycoprotein, TREM1 can bind with a transmembrane receptor DAP12 288 (DNX-activating protein 12) to form a TREM1/DAP12 complex (Lanier, 2009; Ormsby et al., 289 2011; Tessarz \& Cerwenka, 2008). Immunoreceptor tyrosine activation motif (ITAM) tyrosine 290 of DAP12 is phosphorylated by the protein tyrosine kinase of the SRC family, so that the CBL 291 and growth factor receptor binding protein 2 (Grb 2) is phosphorylated. Phospholipid polymyo292 inositol 3 kinase (PI3K) and extracellular signal-regulated kinase (ERK) pathways were further 293 activated, to regulate calcium homeostasis, induce activation of transcription factors, and 294 promote the production of pro-inflammatory cytokines and adhesion molecules (Colonna, 2003; 295 Klesney-Tait et al., 2013; Lanier, 2009; Marco Colonna, 2003; Pelham \& Agrawal, 2014; 296 Turnbull \& Colonna, 2007). TREM1 mainly expressed in myeloid lineage cells, especially in 297 monocytes/macrophages, and it was involved in the innate immune response and amplified 298 proinflammatory response in both infectious and non-infectious diseases (Liu et al., 2019; 299 Pelham \& Agrawal, 2014). TREM1 was closely related to Crohn disease and inflammatory 300 bowel disease (IBD) (Chapuy et al., 2019; Verstockt et al., 2019) and sepsis (Se'bastien Gibot, 301 2004). For sterile inflammatory diseases, it was reported that TREM1 played a critical role in the 302 pathogenesis of Alzheimer's disease (Saadipour, 2017) because of the potential function in 303 innate immune response and DNA methylation (Sao et al., 2018). TREM1 could also predict the 304 prognosis of renal cell carcinoma (Yamada et al., 2018). TREM1-knockout markedly decreased 
305 inflammation and oxidative stress in mice with spinal cord injury, along with the decreased 306 expression of TLR2 and TLR4 (Li et al., 2019). Therefore, potential mechanisms of TREM1 in 307 AMI may be the synthesis of inflammation amplification and the following imbalance of 308 oxidative stress. Although most studies about TREM1 all focused on inflammatory diseases such 309 as IBD, we cannot ignore its role in cardiovascular disease due to emerging immune mechanisms 310 in MI (Pelham \& Agrawal, 2014).

311

312 Univariate analysis showed that TREM1 was positively correlated with BUN, UA, BNP, and LA; 313 and negatively correlated with ALB and ApoA1. As a diagnostic biomarker of heart failure, BNP 314 can be combined with BUN and UA to predict the prognosis of heart failure (Oki et al., 2019; 315 Testani et al., 2014). Increased LA was also associated with the occurrence of AF and acute 316 heart failure (Oikonomou et al., 2019; Van Aelst et al., 2018), while low ALB level was an 317 important predictor for poor prognosis after cardiac surgery (van Beek et al., 2018). ApoA1 was 318 an apolipoprotein of HDL which has anti-inflammatory and antioxidant effects (Gombos et al., 319 2017). TREM1 was related to these markers associated with adverse outcome of cardiac diseases, 320 so we hypothesized that TREM1 may also be associated with the prognosis of myocardial 321 infarction, which was also confirmed by the follow-up results.

322

323 Interestingly, our study showed TREM1 was positively associated with HbAC and FPG; and 324 TREM1 expression was significantly increased in patients with both AMI and diabetes compared 325 to those with only AMI. A study pointed out that sTREM1 expression was significantly higher in 326 patients with obesity and diabetes than those with only obesity or without obesity and diabetes, 327 which points to the important role of TREM1 in the potential pathophysiology of obesity and 328 diabetes (Subramanian et al., 2017). TREM1 overexpression was also related to insulin 329 resistance induced by obesity (Subramanian et al., 2017). A case-control study from Denmark 330 showed sTREM1 level was elevated in children with newly diagnosed type 1 diabetes compared 331 to their siblings (Thorsen et al., 2017). Thus, TREM1 may play an important role in both acute 332 myocardial infarction and diabetes progression.

333

334 We also found that TREM1 was positively related to the number of lesion vessels, although there 335 was no relationship between TREM1 and Gensini score. TREM1 expression in the triple-vessels 336 group was notably higher than other groups. Apart from this, TREM1 could predict six-month 337 MACEs after AMI. It has been reported that TREM1 was involved in the pathogenesis of 338 atherosclerosis. Jeremie Joffre et al used TREM1-knockdown mice and TREM1 inhibitor (LR12 
339

340

341

342

343

344

345

346

347

348

349

350

351

352

353

354

355

356

357

358

359

360

361

362

363

364

365

366

367

368

369

370

371

372

373

peptide) to confirm that TREM1 can promote plaque inflammation and the formation of foam cells, furthermore, TREM1 expression was higher in lipid-rich plaques than that of fibrous plaques (Huynh, 2017; Joffre et al., 2016). High-fat induced high expression of TREM1 in myeloid cells promoted generation of inflammatory factors and formation of foam cells (Zysset $D$, 2016). TREM1 participated in the mechanism of pravastatin improving atherosclerosis (Wang $H M$, 2018). Therefore, increased TREM1 expression may become a potential predictor of vulnerable plaques.

There are still some limitations in this study. First, the follow-up period is only six months and incidence of MACE is low. Second, this study only detected the expression level of TREM1 in the plasma of patients with AMI on admission but did not dynamically monitor the changes of TREM1 one week after myocardial infarction. A follow-up study can focus on the dynamic expression profile of TREM1 after myocardial infarction, to provide a theoretical basis for TREM1 to participate in myocardial infarction risk stratification.

In conclusion, using bioinformatics and after verification using plasma samples of patients with AMI, we found TREM1 is an important biomarker in myocardial infarction. TREM1 was also related to the number of lesion vessels and closely associated with diabetes. TREM1 could also predict the six-month MACEs after AMI. Therefore, TREM1 would be a novel potential predictor for adverse events after MI.

\section{Acknowledgements}

We thank Professor King Lu and Doctor Wang Shiyuan from School of Public Health, Southeast University for statistical support.

\section{References:}

Boufenzer A, Lemarié J, Simon T, Derive M, Bouazza Y, Tran N, Maskali F, Groubatch F, Bonnin P, Bastien C, Bruneval P, Marie PY, Cohen R, Danchin N, Silvestre JS, AitOufella H, Gibot S. 2015. TREM-1 Mediates Inflammatory Injury and Cardiac Remodeling Following Myocardial Infarction. Circulation Research 116(11):1772-1782. doi: 10.1161/CIRCRESAHA.116.305628.

Chapuy L, Bsat M, Sarkizova S, Rubio M, Therrien A, Wassef E, Bouin M, Orlicka K, Weber A, Hacohen N, Villani AC, Sarfati M. 2019. Two distinct colonic CD14(+) subsets characterized by single-cell RNA profiling in Crohn's disease. Mucosal Immunology 12(3):703-719. doi: 10.1038/s41385-018-0126-0. 
374 Colonna M. 2003 TREMs in the immune system and beyond. Nature Reviews Immunology

375

376

377

378

379

380

381

382

383

384

385

386

387

388

389

390

391

392

393

394

395

396

397

398

399

400

401

402

403

404

405

406

407

408

409

410

411

412 3(6):445-453. doi: 10.1038/nri1106.

Gensini GG. 1983. A More Meaningful Scoring System for Determining the Severity of Coronary Heart Disease. American Journal of Cardiology 51:606. doi: 10.1016/s00029149(83)80105-2.

Gombos T, Förhécz Z, Pozsonyi Z, Jánoskuti L, Prohászka Z, Karádi I. 2017. Long-Term Survival and Apolipoprotein A1 Level in Chronic Heart Failure: Interaction With Tumor Necrosis Factor $\alpha$-308 G/A Polymorphism. Journal of Cardiac Failure 23(2):113-120. doi: 10.1016/j.cardfail.2016.06.004.

Huynh K. 2017. Atherosclerosis: TREM1 in atherosclerosis development. Nature Reviews Cardiology 14(2):67. doi: 10.1038/nrcardio.2016.221.

Jeremie L, Amir B, Marc D, Sebastien G. 2015. The Triggering Receptor Expressed on Myeloid cells-1: A new player during acute myocardial infarction. Pharmacological Research 100:261-265. doi: 10.1016/j.phrs.2015.07.027.

Joffre J, Potteaux S, Zeboudj L, Loyer X, Boufenzer A, Laurans L, Esposito B, Vandestienne M, de Jager SC, Hénique C, Zlatanova I, Taleb S, Bruneval P, Tedgui A, Mallat Z, Gibot S, Ait-Oufella H. 2016. Genetic and Pharmacological Inhibition of TREM-1 Limits the Development of Experimental Atherosclerosis. Journal of the American College of Cardiology 68(25):2776-2793. doi: 10.1016/j.jacc.2016.10.015.

Kip KE, Hollabaugh K, Marroquin OC, Williams DO. 2008. The Problem With Composite End Points in Cardiovascular Studies. Journal of the American College of Cardiology 51(7):701-707. doi: 10.1016/j.jacc.2007.10.034.

Klesney-Tait J, Keck K, Li X, Gilfillan S, Otero K, Baruah S, Meyerholz DK, Varga SM, Knudson CJ, Moninger TO, Moreland J, Zabner J, Colonna M. 2013. Transepithelial migration of neutrophils into the lung requires TREM-1. Journal of Clinical Investigation. 123(1):138-149.

Kutikhin AG, Ponasenko AV, Khutornaya MV, Yuzhalin AE, Zhidkova II, Salakhov RR, Golovkin AS, Barbarash OL, Barbarash LS. 2016. Association of TLR and TREM-1 gene polymorphisms with atherosclerosis severity in a Russian population. Meta Gene 9:76-89. doi: 10.1016/j.mgene.2016.04.001.

Lanier LL. 2009. DAP10-and DAP12-associated receptors in innate immunity. Immunological Reveiws 227:150-160. doi: 10.1111/j.1600-065X.2008.00720.x.

Ligthart S, Sedaghat S, Ikram MA, Hofman A, Franco OH, Dehghan A. 2014. EN-RAGE: a novel inflammatory marker for incident coronary heart disease. Arteriosclerosis Thrombosis and Vascular Biology 34(12): 2695-2699. doi: 10.1161/ATVBAHA.114.304306.

Li Z, Wu F, Xu D, Zhi Z, Xu G. 2019. Inhibition of TREM1 reduces inflammation and oxidative stress after spinal cord injury (SCI) associated with HO-1 expressions. Biomedicine Pharmacotherapy 109:2014-2021. doi: 10.1016/j.biopha.2018.08.159. 
413 Liu Q, Johnson EM, Lam RK, Wang Q, Bo Ye H, Wilson EN, Minhas PS, Liu L,

414

415

416

417

418

419

420

421

422

423

424

425

426

427

428

429

430

431

432

433

434

435

436

437

438

439

440

441

442

443

444

445

446

447

448

449

450

451 Swarovski MS, Tran S, Wang J, Mehta SS, Yang X, Rabinowitz JD, Yang SS, Shamloo M, Mueller C, James ML, Andreasson KI. 2019. Peripheral TREM1 responses to brain and intestinal immunogens amplify stroke severity. Nature Immunology 20(8): 1023-1034. doi: 10.1038/s41590-019-0421-2.

Loscalzo J. 2013. Harrison's Cardiovascular Medicine 2th. McGraw-Hill Education.

Marco Colonna FF. 2003. TREM-1 (Triggering Receptor Expressed on Myeloid Cells): A New Player in Acute Inflammatory Responses. The Journalof Infectious Diseases. 187:S397401.

Oikonomou E, Zografos T, Papamikroulis GA, Siasos G, Vogiatzi G, Theofilis P, Briasoulis A, Papaioannou S, Vavuranakis M, Gennimata V, Tousoulis D. 2019. Biomarkers in Atrial Fibrillation and Heart Failure. Current Medicinal Chemistry 26(5): 873-887. doi: 10.2174/0929867324666170830100424.

Oki Y, Kawai M, Minai K, Ogawa K, Inoue Y, Morimoto S, Tanaka T, Nagoshi T, Ogawa T, Yoshimura M. 2019. High Serum Uric Acid is Highly Associated with a Reduced Left Ventricular Ejection Fraction Rather than Increased Plasma B-type Natriuretic Peptide in Patients with Cardiovascular Diseases. Scientific Reports 9(1):682. doi: 10.1038/s41598-018-37053-0.

Ormsby T, Schlecker E, Ferdin J, Tessarz AS, Angelisová P, Köprülü AD, Borte M, Warnatz K, Schulze I, Ellmeier W, Horejsí V, Cerwenka A. 2011. Btk is a positive regulator in the TREM-1/DAP12 signaling pathway. Blood 118(4):936-945. doi: 10.1182/blood-2010-11-317016.

Pelham CJ, Agrawal DK. 2014. Emerging roles for triggering receptor expressed on myeloid cells receptor family signaling in inflammatory diseases. Expert review of clinical immunology 10(2): 243-256. doi: 10.1586/1744666X.2014.866519.

Saadipour K. 2017. TREM1: A Potential Therapeutic Target For Alzheimer's Disease. Neurotoxicity Research 32(1):14-16. doi: 10.1007/s12640-017-9716-y.

Sao T, Yoshino Y, Yamazaki K, Ozaki Y, Mori Y, Ochi S, Yoshida T, Mori T, Iga JI, Ueno SI. 2018. TREM1 mRNA Expression in Leukocytes and Cognitive Function in Japanese Patients with Alzheimer's Disease. Journal of Alzheimers Disease 64(4):1275-1284. doi: 10.3233/JAD-180418.

Gibot S, Kolopp-Sarda MN, Béné MC, Cravoisy A, Levy B, Faure GC, Bollaert PE. 2004. Plasma Level of a Triggering Receptor Expressed on Myeloid Cells-1: Its Diagnostic Accuracy in Patients with Suspected Sepsis. Annals of Internal Medicine 141(1):9-15. doi: 10.7326/0003-4819-141-1-200407060-00009.

Subramanian S, Pallati PK, Rai V, Sharma P, Agrawal DK, Nandipati KC. 2017. Increased expression of triggering receptor expressed on myeloid cells-1 in the population with obesity and insulin resistance. Obesity (Silver Spring) 25(3):527-538. doi: $10.1002 /$ oby. 21714 . 
452

453

454

455

456

457

458

459

460

461

462

463

464

465

466

467

468

469

470

471

472

473

474

475

476

477

478

479

480

481

482

483

484

485

486

487

488

489

490

Subramanian S, Pallati PK, Sharma P, Agrawa DK, Nandipati KC. 2017. TREM-1 associated macrophage polarization plays a significant role inducing insulin resistance in obese population. Journal of Translational Medicine. 15(1):85. doi: 10.1186/s12967-0171187-7.

Tessarz AAS, Cerwenka A. 2008. The TREM-1/DAP12 pathway. Immunology Letters 116(2):111-116. doi: 10.1016/j.imlet.2007.11.021.

Testani JM, Damman K, Brisco MA, Chen S, Laur O, Kula AJ, Tang WH, Parikh C. 2014. A combined-biomarker approach to clinical phenotyping renal dysfunction in heart failure. Journal of Cardiac Failure 20(12):912-919. doi: 10.1016/j.cardfail.2014.08.008.

Thorsen SU, Pipper CB, Mortensen HB, Skogstrand K, Pociot F, Johannesen J, Svensson J; Danish Childhood Diabetes Register. 2017. Levels of soluble TREM-1 in children with newly diagnosed type 1 diabetes and their siblings without type 1 diabetes: a Danish case-control study. Pediatric Diabetes 18(8):749-754. doi: 10.1111/pedi.12464.

Thygesen K, Alpert JS, Jaffe AS, Chaitman BR, Bax JJ, Morrow DA, White HD; Executive Group on behalf of the Joint European Society of Cardiology (ESC)/American College of Cardiology (ACC)/American Heart Association (AHA)/World Heart Federation (WHF) Task Force for the Universal Definition of Myocardial Infarction. 2018. Fourth universal definition of myocardial infarction (2018). Journal of the American College Cardiology 72(18):2231-2264. doi: 10.1016/j.jacc.2018.08.1038.

Turnbull IR, Colonna M. 2007. Activating and inhibitory functions of DAP12. Nature Reviews Immunology 7(2):155-161. doi: 10.1038/nri2014.

Van Aelst LNL, Arrigo M, Placido R, Akiyama E, Girerd N, Zannad F, Manivet P, Rossignol P, Badoz M, Sadoune M, Launay JM, Gayat E, Lam CSP, Cohen-Solal A, Mebazaa A, Seronde MF. 2018. Acutely decompensated heart failure with preserved and reduced ejection fraction present with comparable haemodynamic congestion. European Journal of Heart Failure 20(4):738-747. doi: 10.1002/ejhf.1050.

Van Beek DEC, van der Horst ICC, de Geus AF, Mariani MA, Scheeren TWL. 2018. Albumin, a marker for post-operative myocardial damage in cardiac surgery. Journal of Critical Care 47:55-60. doi: 10.1016/j.jcrc.2018.06.009.

Verstockt B, Verstockt S, Dehairs J, Ballet V, Blevi H, Wollants WJ, Breynaert C, Van Assche G, Vermeire S, Ferrante M. 2019. Low TREM1 expression in whole blood predicts anti-TNF response in inflammatory bowel disease. EBioMedicine 40:733-742. doi: 10.1016/j.ebiom.2019.01.027.

Hu SS, Gao RL, Liu LS, Zhu ML, Wang W, Wang YJ, Wu ZS, Li HJ, Gu DF, Yang YJ, Zheng Z, Chen WW. 2019. Chinese cardiovascular disease report 2018. Chinese Circulation Journal 34(3):209-220.

Wang HM, Gao JH, Lu JL. 2018. Pravastatin improves atherosclerosis in mice with hyperlipidemia by inhibiting TREM-1/DAP12. European Review for Medical and Pharmacological Sciences. 22(15):4995-5003. doi: 10.26355/eurrev_201808_15640. 
491 Wang X, Xu T, Mungun D, Zhou C, Zha Z, Lu M, Fen C, Guo Y. 2019. The relationship between Plasma Soluble Receptor for Advanced Glycation End Products and Coronary Artery Disease. Disease Markers 2019: 4528382. doi: 10.1155/2019/4528382.

Yamada Y, Sugawara S, Arai T, Kojima S, Kato M, Okato A, Yamazaki K, Naya Y, Ichikawa T, Seki N. 2018. Molecular pathogenesis of renal cell carcinoma: Impact of the anti-tumor miR-29 family on gene regulation. International Journal of Urology 25(11):953-965. doi: 10.1111/iju.13783.

Zysset D, Weber B, Rihs S, Brasseit J, Freigang S, Riether C, Banz Y, Cerwenka A, Simillion C, Marques-Vidal P, Ochsenbein AF, Saurer L, Mueller C. 2016. TREM-1 Communications. 7:13151. doi: 10.1038/ncomms13151. 
Figure 1

The flow chart of this study.

\section{Admitted patients}

Meet the first two inclusion criteria and none of the exclusion criteria

Sign informed consent and collect $5 \mathrm{~mL}$ peripheral blood sample

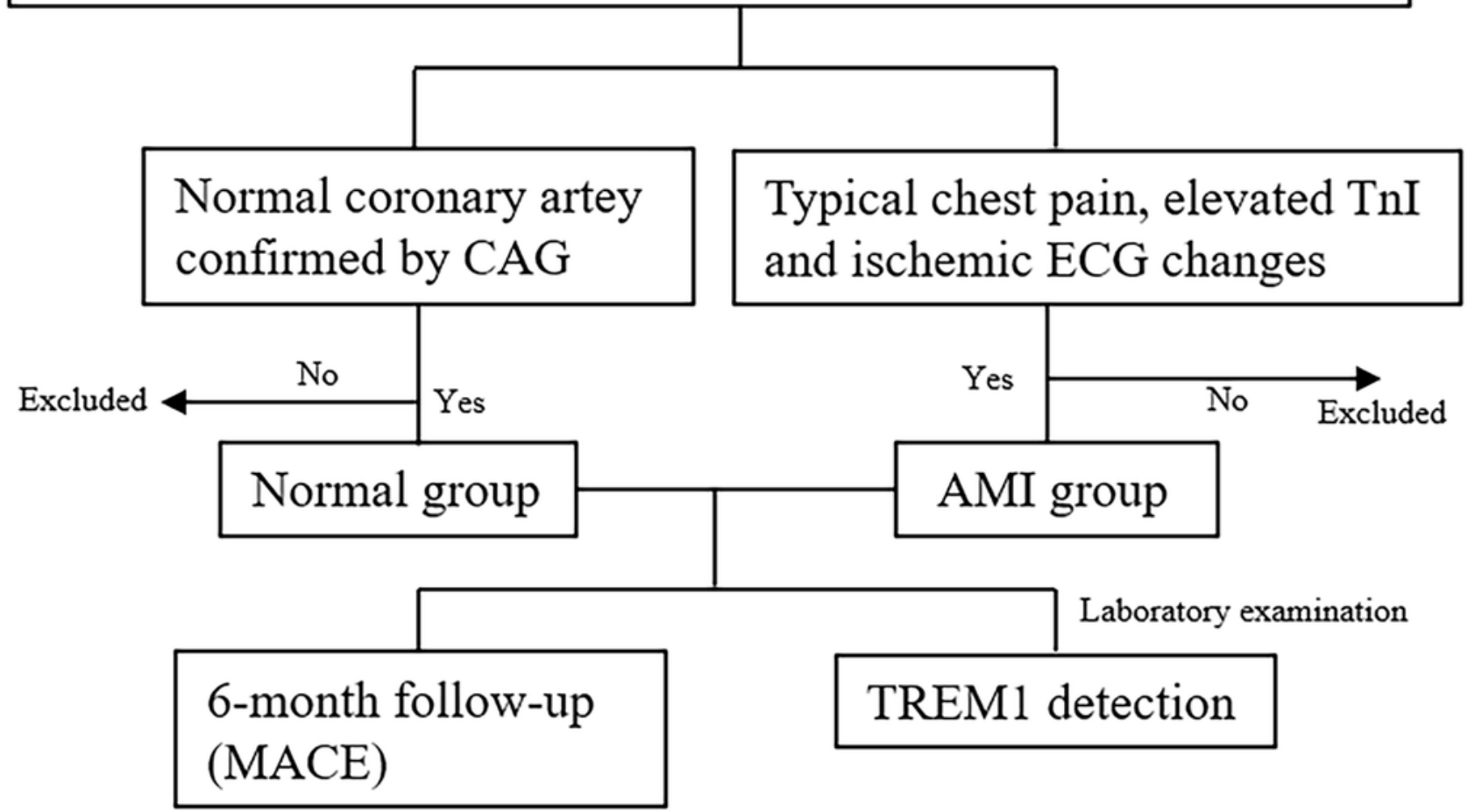




\section{Figure 2}

Candidate genes screening and PPI network construction.

(A/B/C) The volcano plots of GSE66360, GSE60993 and GSE61144 respectively. (D/E) Venn diagram showed 29 upregulated and 3 downregulated common genes were obtained respectively. (F) PPI network of DEGs.

A

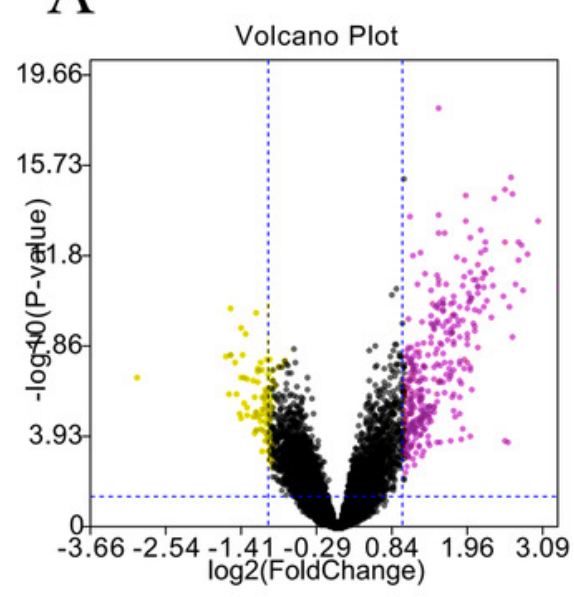

B

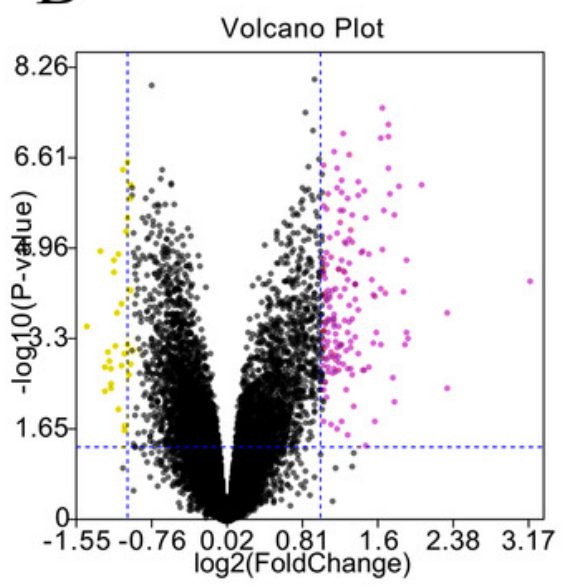

$\mathrm{C}$

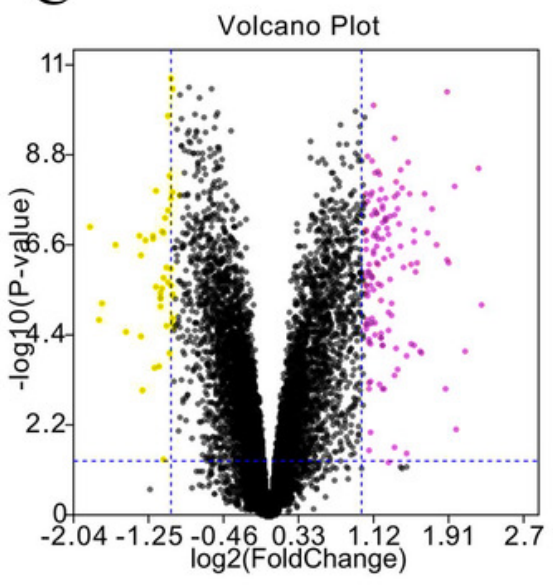

$\mathrm{D}$

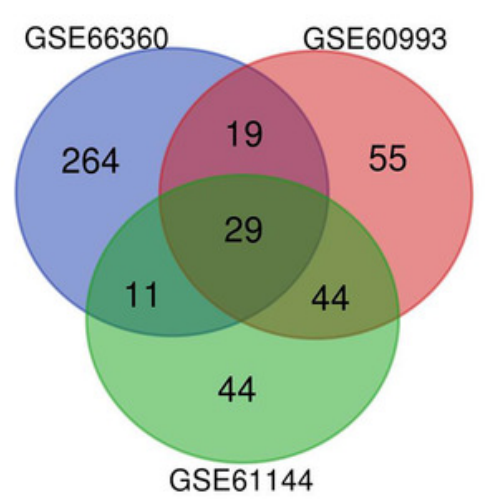

E

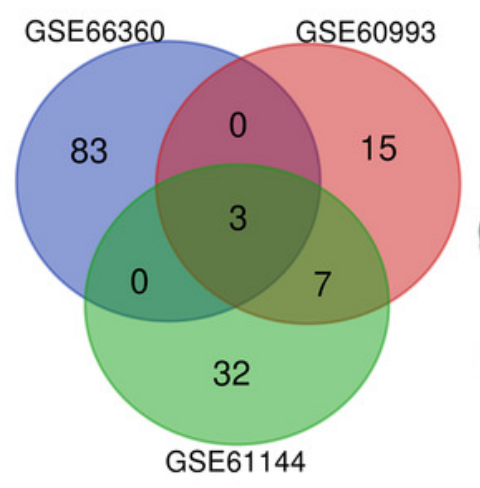

F

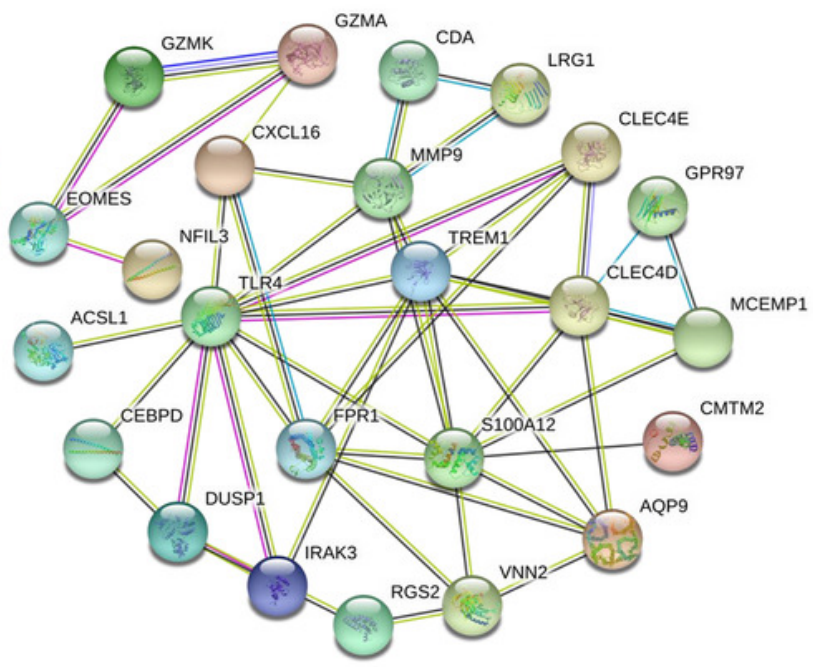


Figure 3

TREM1 expression in different groups.

(A) TREM1 expression in three groups. Compared with the normal group $(140.6 \pm 57.0 \mathrm{pg} / \mathrm{ml}$, $n=35)$, TREM1 expression was markedly increased in AMI group (200.6 $\pm 87.09 \mathrm{pg} / \mathrm{ml}, \mathrm{n}=112$ ) $(p<0.001)$. (B) TREM1 expression in groups with different lesion vessels. TREM1 expression in triple-vessels group $(223.9 \pm 101.0 \mathrm{pg} / \mathrm{ml}, \mathrm{n}=37)$ was significantly higher than that of singlevessel group $(172.5 \pm 74.5 \mathrm{pg} / \mathrm{ml}, \mathrm{n}=36)(P<0.001)$, the level of which in double-vessels group was $202.7 \pm 77.3(\mathrm{pg} / \mathrm{ml}, \mathrm{n}=35)$. (C) TREM1 expression in normal and AMl group with or without diabetes. $* P<0.05, * * P<0.001, \# p=0.06$.

A

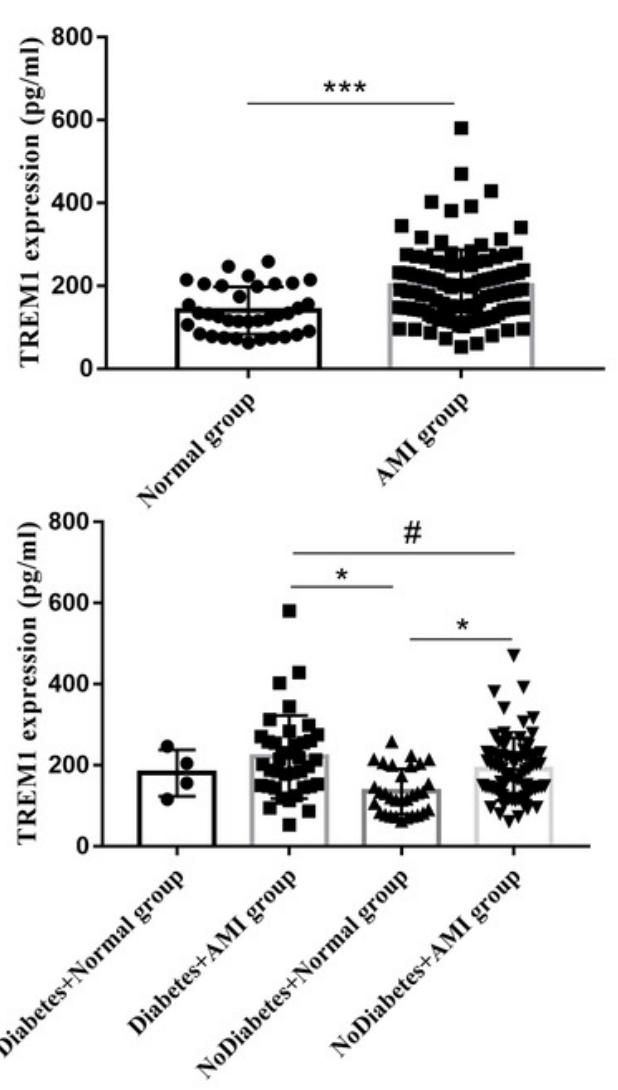

B

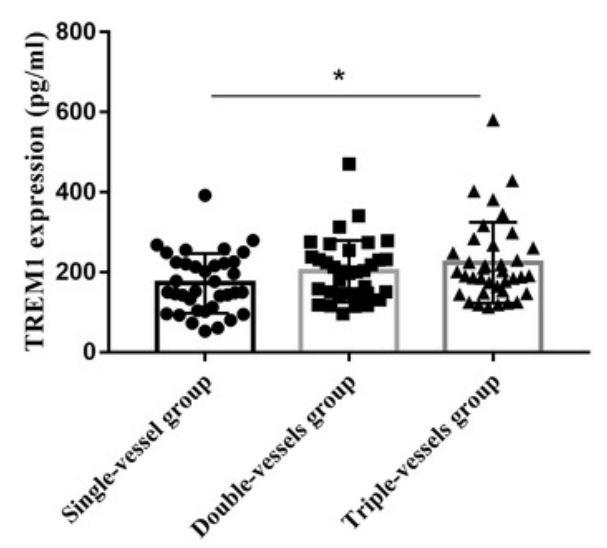


Figure 4

The diagnosis and prognosis value of TREM1 in AMI.

(A) In AMI group $(P<0.001)$, the AUC was 0.721 , with a sensitivity of 0.786 and a specificity of 0.600 (Yoden Index $=0.386$ ). (B) In AMI groups without diabetes, the diagnostic sensitivity was 0.757 , the specificity was 0.631 and the AUC was 0.724 (Yoden Index $=0.402, P<0.001$ ). (C) In Kaplan-Meier analysis, incidence of MACEs in high risk group was obviously higher than that in low risk group $(p<0.0001) .0$, low risk group; 1 , high risk group.
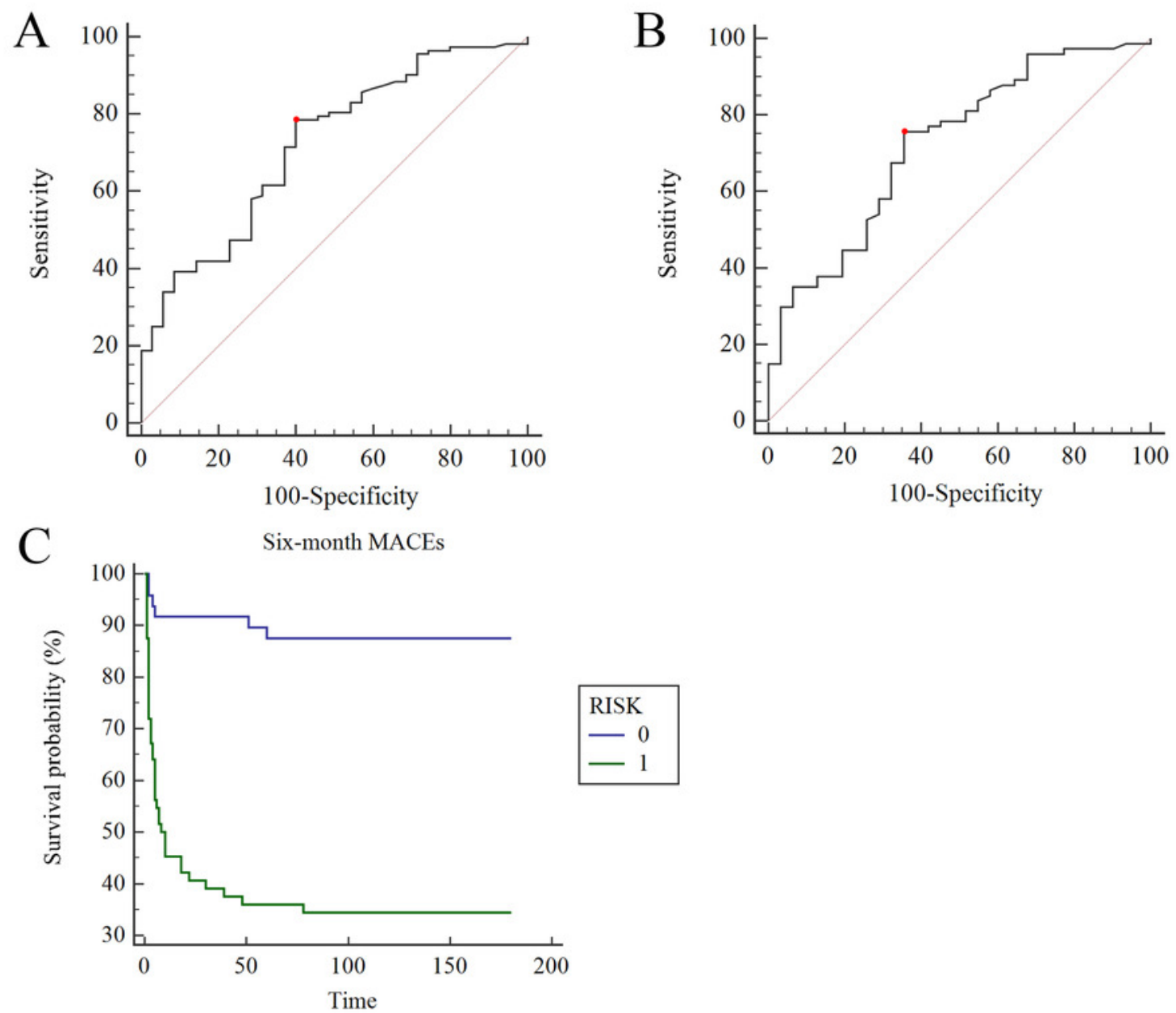
Table $\mathbf{1}$ (on next page)

Common differential expressed genes among GSE66360, GSE61144 and GSE60993. 
29 up-regulated DEGs

TLR4 RGS2 CEBPD CLEC4D AQP9 CMTM2 CPD LRG1

GPR97 PYGL MXD1 DUSP1 IRAK3 DYSF CLEC4E IRS2

S100A12 TREM1 RBP7 VNN2 ACSL1 CXCL16 CDA

CRISPLD2 NFIL3 CYP4F3 MCEMP1 FPR1 MMP9

1

3 down-regulated DEGs

EOMES GZMA GZMK

EOMES GZMA GZMK

(1)

Peer) reviewing PDF | (2021:01:57032:1:1:NEW 14 May 2021) 


\section{Table 2 (on next page)}

Basic characteristics of enrolling patients.

If the data do not satisfy normality and homogeneity of variance ( $S D \geq \chi)$, median and quartile $(25 \%, 75 \%)$ were calculated, otherwise, data was described by $X \pm S D$. $* P<0.05$; $* * \mathrm{P}<0.01$. red blood cells $(\mathrm{RBC})$ and white blood cells $(\mathrm{WBC})$, hemoglobin $(\mathrm{Hb})$, alanine aminotransferase (ALT), aspartate aminotransferase (AST), total cholesterol (TC), total triglyceride (TG), albumin (ALB), Direct bilirubin (DBil), blood urea nitrogen (BUN), uric acid (UA), fasting plasma glucose (FPG), glycosylated hemoglobin (HbAC), C-reactive protein (CRP), triglyceride (TG), total cholesterol (TChol), brain natriuretic peptide (BNP) and left ventricular ejected fraction (LVEF). 


\begin{tabular}{|c|c|c|c|}
\hline Basline characteristics & Normal group $(\mathrm{n}=35)$ & AMI group $(n=112)$ & $\mathrm{P}$ value \\
\hline Age(years) & $54.57 \pm 10.20$ & $61.53 \pm 12.26$ & $0.030^{*}$ \\
\hline Gender(Male/Female) & $12 / 23$ & $83 / 29$ & $0.000^{* *}$ \\
\hline Smoking & 4 & 52 & $0.000^{* *}$ \\
\hline Diabetes & 4 & 38 & $0.010^{*}$ \\
\hline Hypertension & 14 & 70 & $0.033^{*}$ \\
\hline $\mathrm{BMI}(\mathrm{kg} / \mathrm{m} 2)$ & $25.17 \pm 3.70$ & $25.37 \pm 3.51$ & 0.773 \\
\hline \multicolumn{4}{|l|}{ Systolic blood } \\
\hline pressure(mmHg) & $130.43 \pm 14.30$ & $131.25 \pm 20.52$ & 0.826 \\
\hline $\mathrm{WBC}\left(10^{\wedge} 9 / \mathrm{L}\right)$ & $6.16 \pm 1.63$ & $9.72 \pm 3.42$ & $0.000^{* *}$ \\
\hline $\mathrm{RBC}\left(10^{\wedge} 12 / \mathrm{L}\right)$ & $4.61 \pm 0.58$ & $4.41 \pm 0.64$ & 0.108 \\
\hline $\mathrm{Hb}(\mathrm{g} / \mathrm{L})$ & $138.54 \pm 16.67$ & $133.90 \pm 19.65$ & 0.210 \\
\hline $\operatorname{ALB}(\mathrm{g} / \mathrm{L})$ & $40.74 \pm 4.41$ & $38.16 \pm 3.95$ & $0.001^{* *}$ \\
\hline DBil(umol/L) & $3.09 \pm 1.23$ & $4.12 \pm 3.18$ & 0.067 \\
\hline ALT(U/L) & $27.34 \pm 23.29$ & $50.30 \pm 43.11$ & $0.000^{* *}$ \\
\hline AST(U/L) & $23.23 \pm 10.72$ & $136.08 \pm 151.97$ & $0.000^{* *}$ \\
\hline $\mathrm{LDH}(\mathrm{U} / \mathrm{L})$ & $\begin{array}{l}172.00(149.25 \\
200.25)\end{array}$ & $\begin{array}{l}289.50(184.25, \\
598.75)\end{array}$ & - \\
\hline & & 284.00(105.00, & \\
\hline $\mathrm{CK}(\mathrm{U} / \mathrm{L})$ & $74.50(55.5,112.25)$ & 1234.00) & - \\
\hline FPG(mmol/L) & $5.98 \pm 1.96$ & $7.98 \pm 3.37$ & $0.002^{*}$ \\
\hline BUN(mmol/L) & $4.98 \pm 1.26$ & $6.72 \pm 4.91$ & $0.002^{*}$ \\
\hline $\operatorname{Scr}(\mu \mathrm{mol} / \mathrm{L})$ & $66.00(55.00,79.50)$ & $73.00(64.00,86.50)$ & - \\
\hline $\mathrm{UA}(\mu \mathrm{mol} / \mathrm{L})$ & $312.97 \pm 95.56$ & $351.90 \pm 113.14$ & 0.093 \\
\hline $\mathrm{TG}(\mathrm{mmol} / \mathrm{L})$ & $1.62 \pm 0.90$ & $1.69 \pm 1.09$ & 0.743 \\
\hline TChol(mmol/L) & $4.60 \pm 0.93$ & $4.58 \pm 1.08$ & 0.942 \\
\hline $\mathrm{HDL}(\mathrm{mmol} / \mathrm{L})$ & $1.24 \pm 0.33$ & $1.09 \pm 0.24$ & $0.019^{*}$ \\
\hline $\mathrm{LDL}(\mathrm{mmol} / \mathrm{L})$ & $2.78 \pm 0.82$ & $2.80 \pm 0.91$ & 0.918 \\
\hline $\mathrm{HbAC}(\%)$ & $6.02 \pm 1.43$ & $6.78 \pm 1.61$ & $0.047^{*}$ \\
\hline $\mathrm{BNP}(\mathrm{pg} / \mathrm{ml})$ & $20.00(7.00,63.00)$ & $138.50(42.30,530.25)$ & - \\
\hline TnI(ng/ml) & $0.001(0.003,0.01)$ & $1.435(0.017,13.85)$ & - \\
\hline $\mathrm{CRP}(\mathrm{mg} / \mathrm{L})$ & $0.82(0.81,8.02)$ & $7.67(0.82,20.75)$ & - \\
\hline $\operatorname{LVEF}(\%)$ & $0.80 \pm 0.60$ & $0.56 \pm 0.11$ & $0.000^{* *}$ \\
\hline INR & $1.06(1.02,1.09)$ & $1.10(1.05,1.19)$ & - \\
\hline $\operatorname{Ddimer}(\mu \mathrm{g} / \mathrm{L})$ & $71.00(47.00,104.00)$ & $112.50(66.25,232.25)$ & - \\
\hline
\end{tabular}




\section{Table 3 (on next page)}

Pearson and multilinear regression analysis associated with TREM1.

Significant variables with no internal relation in pearson analysis were entered into multilinear analysis, $\mathrm{P}<0.1$ was set for entering variables to avoid losing significant factors. Gensini score representing the severity of CAD was also included in analysis. Methods of "Stepwise", "Forward" and "Backward" in multiple linear regression were all performed by SPSS, which showed that BNP and HbAC were two important independent factors influencing TREM1 expression. Finally, the fitting equation: $Y(T R E M 1)=0.035 * B N P+16.520 * H b A C+66.233$ $(\mathrm{R} 2=0.283, \mathrm{~F}=7.905, \mathrm{P}=0.001) . * \mathrm{P}<0.05 ; * * \mathrm{P}<0.01$. blood urea nitrogen (BUN), uric acid (UA), glycosylated hemoglobin ( $\mathrm{HbAC}$ ), brain natriuretic peptide (BNP), left atrium (LA), albumin (ALB). 


\begin{tabular}{cccccc}
\hline \multirow{2}{*}{ Variables } & \multicolumn{2}{c}{ Pearson analysis } & \multicolumn{3}{c}{ Multilinear analysis } \\
& $\mathrm{r}$ & $\mathrm{p}$ & $\beta$ & $\mathrm{t}$ & $\mathrm{p}$ \\
\hline Age $($ years $)$ & 0.222 & $0.007^{* *}$ & & & \\
Neutrophil $\left(10^{9} / \mathrm{L}\right)$ & 0.209 & $0.011^{*}$ & & & \\
$\mathrm{BUN}(\mathrm{mmol} / \mathrm{L})$ & 0.253 & $0.003^{* *}$ & & & \\
$\mathrm{UA}(\mu \mathrm{mol} / \mathrm{L})$ & 0.335 & $<0.001^{* *}$ & & & \\
$\mathrm{HbAC}(\%)$ & 0.379 & $<0.001^{* *}$ & 16.520 & 1.979 & 0.055 \\
$\mathrm{BNP}(\mathrm{pg} / \mathrm{ml})$ & 0.411 & $<0.001^{* *}$ & 0.035 & 2.922 & $0.006^{* *}$ \\
$\mathrm{LA}(\mathrm{mm})$ & 0.212 & $0.014^{*}$ & & & \\
$\mathrm{ALB}(\mathrm{g} / \mathrm{L})$ & -0.187 & $0.024^{*}$ & & & \\
ApoA1 $(\mathrm{g} / \mathrm{L})$ & -0.167 & $0.046^{*}$ & & & \\
Vessels & 0.244 & $0.011^{*}$ & & & \\
Gensini score & 0.095 & 0.327 & & & \\
\hline
\end{tabular}

1 


\section{Table 4 (on next page)}

Univariate and variate Cox regression analysis for predicting six-month MACEs after AMI.

Significant variables in univariate analysis were entered into multiple Cox regression and only TREM1 was screened out as a significant variable, which indicated that TREM1 was an independent factor predicting six-month MACEs. $* \mathrm{P}<0.05$; $* * \mathrm{P}<0.01$. red blood cells (RBC), hemoglobin $(\mathrm{Hb})$, creatine kinase (CK), blood urea nitrogen (BUN), uric acid (UA), fasting plasma glucose (FPG), left atrium (LA), left ventricular ejected fraction (LVEF). 


\begin{tabular}{ccccccc}
\hline \multirow{2}{*}{ Variables } & \multicolumn{3}{c}{ Unvariate analysis } & \multicolumn{3}{c}{ Variate analysis } \\
& $\operatorname{Exp}(\beta)$ & Wald & $\mathrm{p}$ & $\operatorname{Exp}(\beta)$ & Wald & $\mathrm{p}$ \\
\hline TREM1 $(\mathrm{pg} / \mathrm{ml})$ & 1.007 & 35.535 & $<0.001^{* *}$ & 1.006 & 23.411 & $0.000^{* *}$ \\
Heartrate & 1.029 & 10.479 & $0.001^{* *}$ & & & \\
$\mathrm{RBC}\left(10^{\wedge} 12 / \mathrm{L}\right)$ & 0.656 & 3.896 & $0.048^{*}$ & & & \\
$\mathrm{Hb}(\mathrm{g} / \mathrm{L})$ & 0.984 & 5.543 & $0.019^{*}$ & 0.981 & 7.459 & $0.006^{* *}$ \\
$\mathrm{CK}(\mathrm{U} / \mathrm{L})$ & 1.000 & 5.246 & $0.022^{*}$ & & & \\
$\mathrm{FPG}(\mathrm{mmol} / \mathrm{L})$ & 1.092 & 5.334 & $0.021^{*}$ & & & \\
$\mathrm{BUN}(\mathrm{mmol} / \mathrm{L})$ & 1.052 & 6.216 & $0.013^{*}$ & & & \\
$\mathrm{UA}(\mu \mathrm{mol} / \mathrm{L})$ & 1.002 & 4.049 & $0.044^{*}$ & & & \\
$\mathrm{LA}(\mathrm{mm})$ & 1.874 & 3.826 & 0.050 & & & \\
$\mathrm{IVS}(\mathrm{mm})$ & 1.613 & 4.680 & $0.031^{*}$ & 5.505 & 4.704 & $0.030^{*}$ \\
$\mathrm{LV}(\mathrm{mm})$ & 1.824 & 5.453 & $0.020^{*}$ & & & \\
$\mathrm{LVEF}(\%)$ & 0.013 & 10.849 & $0.001^{* *}$ & & & \\
Vessels & 1.461 & 4.229 & $0.040^{*}$ & & & \\
\hline
\end{tabular}

1 